\title{
0 AGIR DA VIGILÂNCIA SANITÁRIA FRENTE À COVID-19 E 0 NECESSÁRIO EXERCÍCIO DA INTERSETORIALIDADE
}

THE ACTION FROM HEALTH SURVEILLANCE AGAINST COVID-19 AND THE NECESSARY EXERCISE OF INTERSECTORIALITY

Verena Emmanuelle Soares Ferreira ${ }^{1}$

Jéssica Maria Cavalcante Mesquita 2

Pollyana Dias Parente ${ }^{3}$

Luiz Galdino da Costa Filho ${ }^{4}$

Marília Gouveia Ferreira Lima ${ }^{5}$

Alex Melo de Aguiar 6

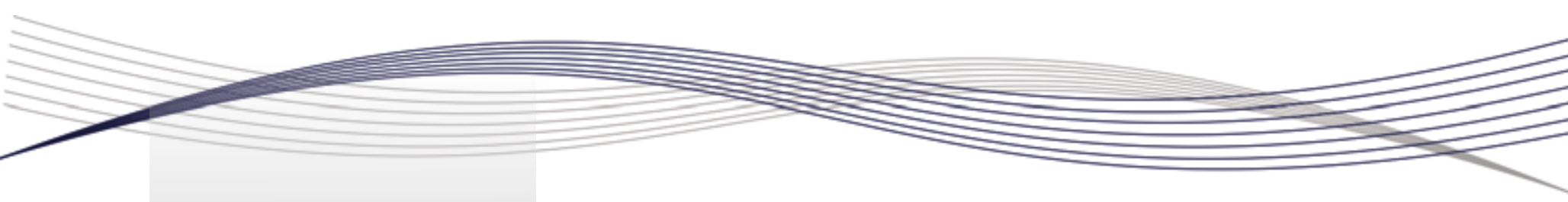

Palavras-chave:

Vigilância Sanitária; Infecções por Coronavirus; Intersetorialidade.

Keywords: Health Surveillance; Coronavirus Infections; Intersectoriality.

Palabras clave: Vigilancia Sanitaria; Infecciones por Coronavirus; Intersectorialidad.

Submetido: $10 / 11 / 20$

Aprovado: 28/12/20

Autor(a) para Correspondência: Verena Emmanuelle Soares Ferreira Vila Cisne, 102 - Pedrinhas Sobral - CE CEP: $62.040-770$ E-mail:verenaemmanuelle@gmail.com

\section{RESUMO}

O trabalho da Vigilância Sanitária se insere no espaço regulatório do Estado, com atribuições indelegáveis de controle sanitário de processos, ambientes e da produção e consumo de bens e serviços de interesse da saúde, assumindo um importante papel na prevenção de agravos e promoção da saúde. Este estudo tem como objetivo descrever as principais ações de vigilância sanitária no curso da pandemia, no município de Sobral-CE. Tratase de estudo exploratório descritivo, do tipo relato de experiência, com abordagem qualitativa, realizado em Sobral, Ceará, de março a setembro de 2020. Os cinco principais eixos de ações priorizados pela Vigilância Sanitária no curso da pandemia foram: (I) gestão e planejamento; (II) controle do risco sanitário; (III) regulação; (IV) monitoramento dos casos suspeitos e confirmados de Covid-19 em descumprimento do isolamento social; e (V) informação, comunicação e educação para a saúde, que necessitaram de articulações complexas para a sua execução e efetividade. Ações, essas, de natureza regulatória e fundamentalmente intersetoriais. A experiência reforça a importância de novas estratégias que constituam iniciativas voltadas para a superação de desafios históricos no processo de trabalho da Vigilância Sanitária, tornando o fazer mais integral e resolutivo, propondo uma articulação de políticas intersetoriais voltadas à melhoria da saúde coletiva.

\footnotetext{
1. Enfermeira. Mestra em Saúde da Família (UFC). Gerente da Célula de Vigilância Sanitária. Secretaria da Saúde de Sobral. Prefeitura de Sobral. E-mail: verenaemmanuelle@gmail.com ORCID: https://orcid.org/0000-0002-3714$\underline{3406}$

2. Médica Veterinária. Técnica da Vigilância Sanitária de Sobral. Secretaria de Saúde de Saúde. E-mail: jesca_ mcm@hotmail.com 0RCID: https://orcid.org/0000-0001-9056-857X

3. Tecnóloga de Alimentos. Especialista em Vigilância Sanitária de Alimentos. Técnica da Vigilância Sanitária de Sobral. Secretaria de Saúde de Saúde. E-mail: pollyanadp@hotmail.com ORCID: https://orcid.org/0000-00033569-7138

4. Farmacêutico. Supervisor do Núcleo de Fiscalização de Produtos e Serviços de Interesse a Saúde. Vigilância Sanitária. Secretaria de Saúde de Saúde. E-mail: galdino.filho@sobral.ce.gov.br 0RCID: https://orcid.org/00000002-0826-2529

5. Arquiteta Urbanista. Mestra em Engenharia de Transportes (UFC). Secretária do Urbanismo e Meio Ambiente. Prefeitura de Sobral. E-mail: mariliaflima@sobral.ce.gov.br 0RCID: https://orcid.org/0000-0003-3334-7062

6. Engenheiro Civil. Coordenador de Vistoria e Fiscalização da SEUMA. Prefeitura de Sobral. E-mail: alexmelo@ sobral.ce.gov.br ORCID: https://orcid.org/0000-0002-4311-0440
} 


\section{ABSTRACT}

The work of the Health Surveillance is inserted in the regulatory space of the State, with non-delegable attributions of sanitary control of processes, environments, and the production and consumption of goods and services of health interest, assuming an important role in disease prevention and health promotion. This study aims to describe the main health surveillance actions in the course of the pandemics, in the municipality of Sobral-CE. Descriptive exploratory, experience report type of study, with a qualitative approach, carried out in Sobral-CE, from March to September 2020. The five main axes of actions prioritized by Health Surveillance in the course of the pandemics were: (I) management and planning; (II) health risk control; (III) regulation; (IV) monitoring of Covid-19 suspected and confirmed cases in breach of social isolation; and (V) information, communication, and health education, which required complex articulations for its execution and effectiveness. These actions are of a regulatory nature and fundamentally intersectoral. The experience reinforces the importance of new strategies, which are initiatives aimed at overcoming historical challenges in the health surveillance work process, making the strategies more comprehensive and decisive, proposing an articulation of intersectoral policies aimed towards improving public health.

\section{RESUMEN}

El trabajo de la Vigilancia Sanitaria se inserta en el espacio regulatorio del Estado, con atribuciones indelegables de control sanitario de procesos, ambientes y de la producción y consumo de bienes y servicios de interés de la salud, asumiendo un importante papel en la prevención de enfermedades y promoción de la salud. Este estudio tiene como objetivo describir las principales acciones de vigilancia sanitaria en el curso de la pandemia, en el municipio de Sobral-CE. Estudio exploratorio descriptivo, del tipo relato de experiencia, con enfoque cualitativo, realizado en Sobral-CE, de marzo hasta septiembre de 2020. Los cinco principales ejes de acciones priorizados por la Vigilancia Sanitaria en el curso de la pandemia han sido: (I) gestión y planeamiento; (II) control de riesgo sanitario; (III) regulación; (IV) monitoreo de los casos sospechosos y confirmados de Covid-19 en incumplimiento del aislamiento social; y (V) informaciones, comunicación y educación para la salud, que necesitaron de articulaciones complejas para la ejecución y afectividad. Acciones, esas, de naturaleza regulatoria y fundamentalmente intersectoriales. La experiencia refuerza la importancia de las nuevas estrategias que constituyen esfuerzos para la superación de desafíos históricos en el proceso de trabajo de la vigilancia sanitaria, volviendo el hecho de hacer más integral y resolutivo, proponiendo una articulación de políticas intersectoriales vueltas a la mejoría de la salud colectiva.

\section{INTRODUÇÃO}

Em todas as épocas ocorreram intervenções do Poder de Autoridade sobre as práticas de cura, os medicamentos, os alimentos, a água e o ambiente. Com o avanço das forças produtivas, surgiram intervenções sobre a circulação dos meios de transporte, cargas e pessoas, bem como sobre o consumo da força de trabalho, mediante distintas formas de regulação e intervenção nas práticas do mercado. Estabeleceram-se regras para o exercício de atividades relacionadas a tais elementos, visando proteger a saúde das pessoas e da coletividade. As regras acompanham o desenvolvimento científico e tecnológico e a organização do poder nas sociedades, que se apresentam de formas e graus diferenciados ${ }^{1}$.

0 escopo de competências e os modelos organizacionais e operacionais não são idênticos entre os países, tampouco a identificação. A denominação Vigilância Sanitária (VISA) foi adotada no Brasil, mas não existe um termo universal para nominar a área. A definição incorporada à Lei Orgânica da Saúde (Lei 8.080, art. $6^{\circ}$, parágrafo $1^{\circ}$ ) delineia um marco referencial de natureza preventiva e do âmbito das relações sociais de produção-consumo, como:

[...] um conjunto de ações capaz de eliminar, diminuir ou prevenir riscos à saúde e de intervir nos problemas sanitários decorrentes do meio ambiente, da produção e circulação de bens e da prestação de serviços de interesse da saúde, abrangendo o controle de bens de consumo que, direta ou indiretamente, se relacionem com a saúde, compreendidas todas as etapas e processos, da produção ao consumo; o controle da prestação de serviços que se relacionam direta ou indiretamente com a saúde ${ }^{2}$. 
Para Costa, esse conceito conferiu à VISA uma reformulação do seu papel, para além das suas antigas práticas policialescas, ampliando sua área de atuação, da prevenção de agravos e proteção da saúde, e exigiu também dos seus profissionais um conhecimento sanitário amplo e embasado nos referenciais de risco, da qualidade e da segurança ${ }^{3}$.

0 trabalho da VISA apresenta especificidades devido à natureza dos objetos de intervenção e do caráter exclusivamente estatal e disciplinador de suas ações. Os seus objetos de intervenção podem ser entendidos como objetos complexos, que se situam no espaço de relações entre a ciência, a saúde e o mercado. As especificidades dos processos de trabalho da VISA a distinguem do trabalho em saúde em geral e a insere no espaço regulatório do Estado, com atribuições indelegáveis de controle sanitário de processos, de ambientes e da produção e consumo de bens e serviços de interesse da saúde ${ }^{4}$.

Em função da sua diversidade de objetos e das práticas que executa, a VISA vem sendo considerada uma das faces mais complexas da saúde pública, já que atua em um campo de articulação entre os domínios econômico, jurídico-político e médico-sanitário, além de englobar atividades de natureza interdisciplinar, multiprofissional e interinstitucional ${ }^{3}$.

Uma série de desafios surge diuturnamente para a VISA, sendo o mais recente ocasionado pela pandemia de Covid-19, doença causada pelo novo coronavírus, também denominado SARS-CoV-2 (severe acute respiratory syndrome coronavirus 2 ). 0 enfrentamento desses desafios, além da execução das atividades rotineiras, exige a implementação de ferramentas de gestão e a transformação dos processos executados pela VISA.

A Covid-19 foi identificada pela primeira vez na China, em dezembro de 2019. Em 30 de janeiro de 2020, a Organização Mundial da Saúde (OMS) declarou que a epidemia de Covid-19 constituía uma Emergência de Saúde Pública de Importância Internacional (ESPII) e, em 11 de março de 2020, uma pandemia ${ }^{5}$. Diante da constatação de que as estratégias adotadas não eram suficientes para conter a proliferação da doença, medidas mais severas de Saúde Pública, incluindo o diagnóstico oportuno, o isolamento dos casos e a quarentena nas comunidades, foram recomendadas pela OMS aos países ${ }^{6}$.

Para Porto, a crise atual faz parte de uma crise civilizatória mais ampla e com múltiplas dimensões - social, econômica, democrática, ambiental e

\section{"A crise atual faz parte \\ de uma crise civilizatória \\ mais ampla e com múltiplas \\ dimensões - social, \\ econômica, democrática, ambiental e sanitária"}

sanitária - de modo que a saúde pública/coletiva precisará se reinventar ${ }^{7}$. Com isso, a Vigilância Sanitária, assim como as outras vigilâncias, caracteriza-se como parte da capacidade de resposta do Sistema Único de Saúde (SUS) a um evento como esse e foi surpreendida com novas demandas para algumas das quais não estava preparada, assim como todo o SUS.

No mês de março, o município de Sobral publicou decretos reconhecendo o estado de emergência em âmbito municipal, estabelecendo medidas para o enfrentamento do novo coronavírus, assim como instituiu o Plano Municipal de Contingência para Infecção Humana pelo Novo Coronavírus ${ }^{8,9}$.

Os desafios colocados para toda a estrutura do Sistema de Saúde foram inúmeros, dentre os principais, apontam-se: a necessidade de dar respostas rápidas, com alta frequência, para questões complexas; a regulamentação de diversos temas em tempo recorde; a ausência de evidência científica para apoiar a tomada de decisão; a mudança nos processos e nas rotinas de trabalho; os conflitos e as resistências por parte da população e do setor regulado; as pressões políticas e econômicas que colidem com o interesse público; o número insuficiente de profissionais; e o planejamento e execução de ações intra e intersetoriais.

Diante do exposto, este artigo tem como objetivo descrever as principais ações de Vigilância Sanitária no curso da pandemia, exaltando o permanente exercício da intersetorialidade no município de Sobral-CE.

\section{METODOLOGIA}

Trata-se de estudo exploratório descritivo, do tipo relato de experiência, com abordagem qualitativa, realizado no período de março a setembro de 2020, durante o enfrentamento da pandemia de Covid-19, em Sobral, município localizado na zona norte do estado do Ceará. 0 município está localizado na 
porção noroeste do estado, a $240 \mathrm{~km}$ da capital Fortaleza, por via da BR-222. A cidade, que conta com uma área territorial de $2.122,98 \mathrm{~km}^{2}$, possui a quinta maior população do estado, sendo estimada, para 2020, em 210.711 habitantes, concentrando-se a maior parte na zona urbana ${ }^{10}$. Destaca-se ainda que está localizada no sertão e possui clima tropical, quente e seco.

o Sistema de Saúde de Sobral conta com uma ampla rede de serviços que perpassam os vários níveis de atenção à saúde e se configuram como referência para a macrorregião norte do Ceará. 0s indicadores de saúde oriundos dessa rede complexa e dos processos nela desenvolvidos têm merecido reconhecimento.

De acordo com Soares e Linhares, o município de Sobral foi certificado, em janeiro de 2001, para assumir a gestão das Ações de Epidemiologia e Controle de Doenças, de acordo com as competências e a sistemática de financiamento definidas na Portaria n. ${ }^{\circ} 1399 / \mathrm{GM}$, de 15 de dezembro de $1999^{11}$. Com essa medida, o município passou a ser responsável, perante o Ministério da Saúde (MS), por todas as ações que se referem às áreas de vigilância, prevenção e controle de doenças. Com a organização e a municipalização do SUS, desencadeadas em 1997, foi implantada a Coordenação de Vigilância à Saúde. Na estruturação da Vigilância à Saúde de Sobral, inicialmente foram integradas as ações nas áreas da Vigilância Epidemiológica, Vigilância Sanitária, Vigilância Nutricional e Vigilância dos Fatores Biológicos de Risco, sendo essas as bases de operação das ações do Centro de Controle de Zoonoses $(C C Z)^{12}$.

0 atual organograma da Secretaria de Saúde de Sobral traz, na estruturação da Coordenadoria de Vigilância em Saúde, sete células, a saber: Célula do Centro de Referência em Saúde do Trabalhador (CEREST); Célula de Vigilância Epidemiológica (VIGEP); Célula de Imunização; Célula da Unidade de Vigilância de Zoonoses (UVZ); Célula de Vigilância em Saúde Ambiental (VISAM); Célula de Vigilância Alimentar e Nutricional (VAN); e Célula de Vigilância Sanitária (VISA), que tem o Núcleo de Fiscalização de Produtos e Serviços de Interesse à Saúde ${ }^{13}$.

Com 0 estabelecimento das medidas mais restritivas para o enfrentamento da Covid-19 e a instituição do plano municipal de contingência, foi necessária a tomada de decisões rápidas e ações integradas para o seu contingenciamento. A VISA foi um dos elementos principais para a elaboração

\section{"As ações de vigilância sanitária permitiram, durante a pandemia, o fortalecimento das articulações intrasetoriais e intersetoriais"}

de respostas diante da pandemia. Iniciou-se uma articulação entre as Células de Vigilância em Saúde para intensificar as ações de fiscalização, buscandose averiguar a adoção das medidas e orientação ao setor regulado, trabalhadores e a população sobre os cuidados de prevenção e controle da infecção.

Partindo desses movimentos, o presente artigo sistematiza a experiência em cinco seções correspondentes aos cinco principais eixos de ações priorizados pela VISA no curso da pandemia: (I) Gestão e planejamento; (II) Controle do Risco Sanitário; (III) Regulação; (IV) Monitoramento dos casos suspeitos e confirmados de Covid-19 em descumprimento do isolamento social e (V) Informação, comunicação e educação para a saúde.

\section{RESULTADOS E DISCUSSÃO}

As ações de vigilância sanitária permitiram, durante a pandemia, o fortalecimento das articulações intrasetoriais e intersetoriais e promoveram discussões bastante significativas sobre os processos de trabalho. Dentre os órgãos que efetivamente realizaram ações conjuntas, citamos as Células de Vigilância em Saúde e a Escola de Saúde Pública Visconde de Saboia (ESP-VS), assim como a Secretaria de Urbanismo e Meio Ambiente (SEUMA), a Autarquia Municipal de Meio Ambiente (AMMA), a Guarda Civil Municipal de Sobral (GCMS) e a Polícia Militar do Ceará (PMCE).

0 tema da Vigilância Sanitária integra o campo da Saúde Coletiva e pode ser compreendido como um segmento específico do sistema público de saúde, de articulações complexas de natureza jurídicopolítica, econômica e médico-sanitária, tendo a sua prática sócio-histórica estruturada pelo Estado para a proteção e a defesa da saúde. Suas ações, de natureza regulatória e fundamentalmente intersetoriais, perpassam as relações entre ciência, mercado, saúde e sociedade ${ }^{14}$.

As narrativas vividas pelos autores e as sínteses 
produzidas serão desenvolvidas nas seções a seguir, considerando os cinco eixos delineados como prioritários de atuação no curso da pandemia de Covid-19.

\section{Gestão e planejamento}

Com um aparato de conhecimentos, funções e instrumentos, a Vigilância Sanitária atua principalmente por meio de regulamentações sobre a concessão de licenças sanitárias para a produção e o comércio de bens e serviços; registro de produtos para a fabricação e consumo; certificação de boas práticas de produção; monitoramento da qualidade de produtos e serviços; fiscalização do cumprimento das normas; comunicação e educação sobre os riscos; e a vigilância de eventos adversos relacionados a esses bens ${ }^{15}$.

Dentre as ações de Gestão e Planejamento, a VISA de Sobral, Ceará, realizou o mapeamento das principais atividades produtivas do município e os serviços classificados como essenciais, para que fossem devidamente vistoriados; além disso, delineou-se o estabelecimento de metas de cobertura e a necessidade de orientação a serem realizados para empregadores, trabalhadores e a população. Articulou-se a organização de trabalhos de forma ininterrupta, incluindo feriados e finais de semanas. Nos meses de março e abril, foram realizadas reuniões semanais com os gerentes das Células de Vigilância em Saúde do Trabalhador, Vigilância em Saúde Ambiental, Unidade de Vigilância de Zoonoses, Vigilância Alimentar e Nutricional e Vigilância Sanitária para o planejamento conjunto entre as vigilâncias, com a identificação de prioridades para a atuação integrada, além de produzir e disponibilizar informações importantes para a tomada de decisão.

Vale ressaltar a participação periódica nas reuniões com a gestão municipal para a tratativa sobre o planejamento e as discussões do processo de flexibilização e deliberações relacionadas ao processo de reabertura.

\section{Controle Sanitário}

0 controle sanitário, relacionado às fiscalizações sanitárias e aos processos administrativos sanitários deflagrados a partir de irregularidades identificadas nas inspeções e/ou acolhidas por meio de denúncias, constituiu-se enquanto um dos maiores desafios vivenciados no curso da pandemia. 0 Gráfico 1 , a seguir, apresenta o número de fiscalizações realizadas pela VISA no curso dos meses de março a setembro.

Gráfico 1 - Fiscalizações realizadas pela Vigilância Sanitária de Sobral entre os meses de março e setembro de 2020 .

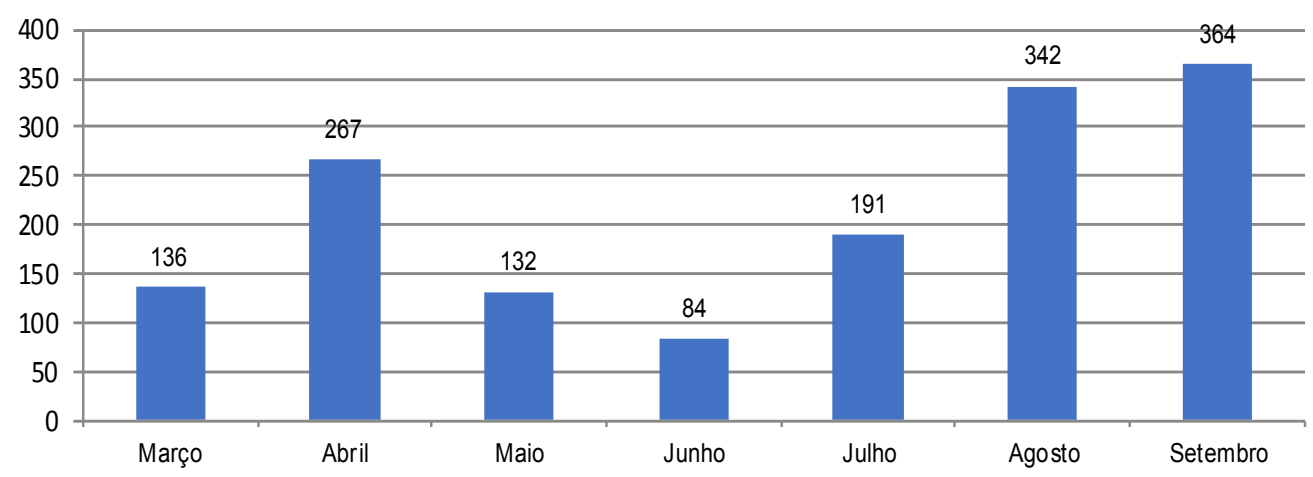

Fonte: VISA/SMS/PMS.

Nota-se, a partir do gráfico apresentado, que foram realizadas, no período de sete meses, mais de 1.500 fiscalizações, com uma tendência a crescimento nos meses de agosto e setembro. Com a fase de retomada da economia a partir do mês de julho, as ações se ampliaram. A fiscalização sanitária é um dos momentos de concreção do exercício do poder que detém o Estado para aceitar ou recusar produtos, bens, processos, 
tecnologias ou serviços sob o controle sanitário e para intervir em situações de risco à saúde. A fiscalização verifica o cumprimento das normas estabelecidas, sendo, portanto, o corolário da legislação $0^{14}$.

Costa e Kobayashicio afirmam que, durante as inspeções, o profissional da VISA deve compreender a finalidade e o funcionamento dos equipamentos para poder detectar se estão ou não corretos os procedimentos operacionais em cada situação vivenciada. Um erro cometido durante a realização de um processo pode colocar a saúde da coletividade em risco. Ao detectar as possíveis falhas de um procedimento ou de um equipamento durante uma inspeção, o profissional da VISA cumpre o seu papel de identificar, minimizar e prevenir riscos à saúde do usuário.

Ao mesmo tempo em que era requisitada maior atuação da VISA, havia uma redução no número de fiscais sanitários, resultado do afastamento de profissionais que fazem parte do grupo de risco para a Covid-19. Para a superação desse descompasso, a cooperação intra e intersetorial foi fundamental para o alcance dos resultados esperados. A Política Nacional da Promoção da Saúde refere-se à intersetorialidade como o processo de articulação de saberes, potencialidades e experiências de sujeitos, grupos e setores na construção de intervenções compartilhadas, estabelecendo vínculos, corresponsabilidade e cogestão para objetivos comuns ${ }^{17}$.

As demandas emergidas nesse processo apontaram a necessidade da realização de fiscalizações conjuntas, e a equipe de vistoria e fiscalização da Secretaria de Urbanismo e Meio Ambiente se mostrou essencial para o fortalecimento e maior efetividade das ações, criando e ampliando espaços interinstitucionais de discussão e negociação que convergiram para uma nova prática sanitária.

Dentre as principais irregularidades encontradas durante as fiscalizações, registraramse: o funcionamento de estabelecimentos não autorizados; os aspectos relacionados à organização e higiene e a ausência de medidas de prevenção e controle de infecção pelo novo coronavírus, como o distanciamento recomendado, barreiras de proteção, prática de lavagem das mãos, higienização das mãos com álcool $70 \%$, etiqueta respiratória, equipamento de proteção individual (EPI) ausente, incompleto ou inadequado à atividade.

Vale apontar o importante monitoramento das

\section{"A cooperação intra e intersetorial foi fundamental para o alcance dos resultados esperados"}

gestões municipal e estadual para as instituições de longa permanência para idosos (ILPI). Desde a publicação do primeiro decreto do município, houve a restrição de acesso e a suspensão de visitas aos locais. A Vigilância realizou a inspeção e o monitoramento mensal. Moraes ${ }^{18}$ et al. afirmam que as ILPI representam o principal fator de risco para morbimortalidade da infecção pelo novo coronavírus, pois reúnem todas as condições necessárias para a "tempestade perfeita". Em países da América do Norte, Ásia, Europa e 0ceania, entre $30 \%$ e $60 \%$ de todos os óbitos pela Covid-19 ocorreram nas ILPI e afins. E, no município de Sobral, não houve registros de óbitos pela Covid-19 em ILPI.

Outra estratégia adotada pelo município para o controle sanitário tratou-se das barreiras sanitárias, que tiveram como objetivo restringir a circulação de transportes e pessoas para prevenir o risco de contaminação e disseminação da Covid-19. As barreiras foram executadas pela Secretaria de Segurança e Cidadania, por meio da Guarda Civil Municipal de Sobral (GCMS). Nas entradas e saídas do município, os agentes da GCMS realizavam abordagem aos condutores que precisavam entrar na cidade; caso o motorista não estivesse se deslocando por um motivo essencial, ele seria convidado a retornar ao seu local de origem. Nos casos de maior complexidade, os técnicos da VISA eram acionados para orientações, averiguação e condutas necessárias.

Os ônibus que traziam trabalhadores vindos de outros estados para prestar serviços em atividades classificadas como essenciais eram autorizados a entrar no município após a GCMS e a Coordenadoria Municipal de Trânsito acionar a VISA para 0 monitoramento desses trabalhadores. Os técnicos da VISA realizavam uma triagem com a aferição de temperatura, entrevista pontuando possiveis sinais e sintomas relacionados à infecção pelo novo coronavírus e a condução até a rede de hotelaria para a avaliação das condições e medidas adotadas pelo respectivo setor, o que resultou em notificação 
de três hotéis por irregularidades e mais de 90 trabalhadores abordados durante o período de lockdown do município.

\section{Regulação}

No que diz respeito à regulação, a Vigilância Sanitária atuou em vários contextos. As atividades ocorreram de forma mais intensa no credenciamento de farmácias e laboratórios para a testagem da Covid-19 e no acompanhamento junto ao Ministério Público (MP) para que as informações dos resultados dos testes de detecção de Covid-19 fossem repassadas diariamente à Secretaria de Saúde do Município.

0 processo de elaboração e atualização do marco normativo relacionado à flexibilização das atividades econômicas no município, assim como a estruturação e a organização dos protocolos sanitários, geral e setoriais, que, até novembro, totalizavam mais de 30 protocolos, é fruto de intensas discussões entre os profissionais, análise de contexto e levantamento de dados em bases científicas, na busca de material qualificado.

Nesse contexto, observa-se que a Vigilância e a Regulação Sanitárias têm como função mediar os interesses de saúde e econômicos, de modo que se reportam à avaliação e ao gerenciamento de riscos e incertezas relacionados aos objetos que a sociedade define como de interesse da saúde. As normas são fundamentais, mas não encerram as possibilidades de intervenção para proteger a saúde. Em situações de risco iminente não normatizadas, podem ser acionados princípios e medidas acautelatórios em defesa da saúde ${ }^{14}$.

Em $1^{\circ}$ de julho de 2020, a fase de transição no município e as liberações de alguns setores da economia foram iniciadas. De acordo com o Plano de Retomada ${ }^{19}$, as empresas autorizadas a funcionar na fase de transição, assim como nas fases subsequentes, necessitaram emitir um certificado de reabertura, documento obrigatório a ser colocado na entrada do estabelecimento, juntamente com o protocolo de medidas sanitárias, geral e setorial, a ser adotado pelo setor.

Todos os certificados de reabertura disponibilizados de acordo com cada fase de flexibilização possuem um QR code (Quick Response) de segurança para a garantia da veracidade do documento pela Vigilância Sanitária, que, durante as fiscalizações, verifica a validação e o compromisso do setor regulado em cumprir os protocolos. Durante a abordagem dos estabelecimentos, verificava-se o certificado de reabertura, a implementação dos protocolos, o preenchimento do checklist, a emissão do laudo de inspeção e, no caso de atendimento das medidas, o estabelecimento recebe um selo emitido pela VISA (Figura 1 e 2).

Figura 1 - Registro de inspeção, certificado de reabertura e selo de cumprimento das medidas sanitárias. Sobral, 2020.
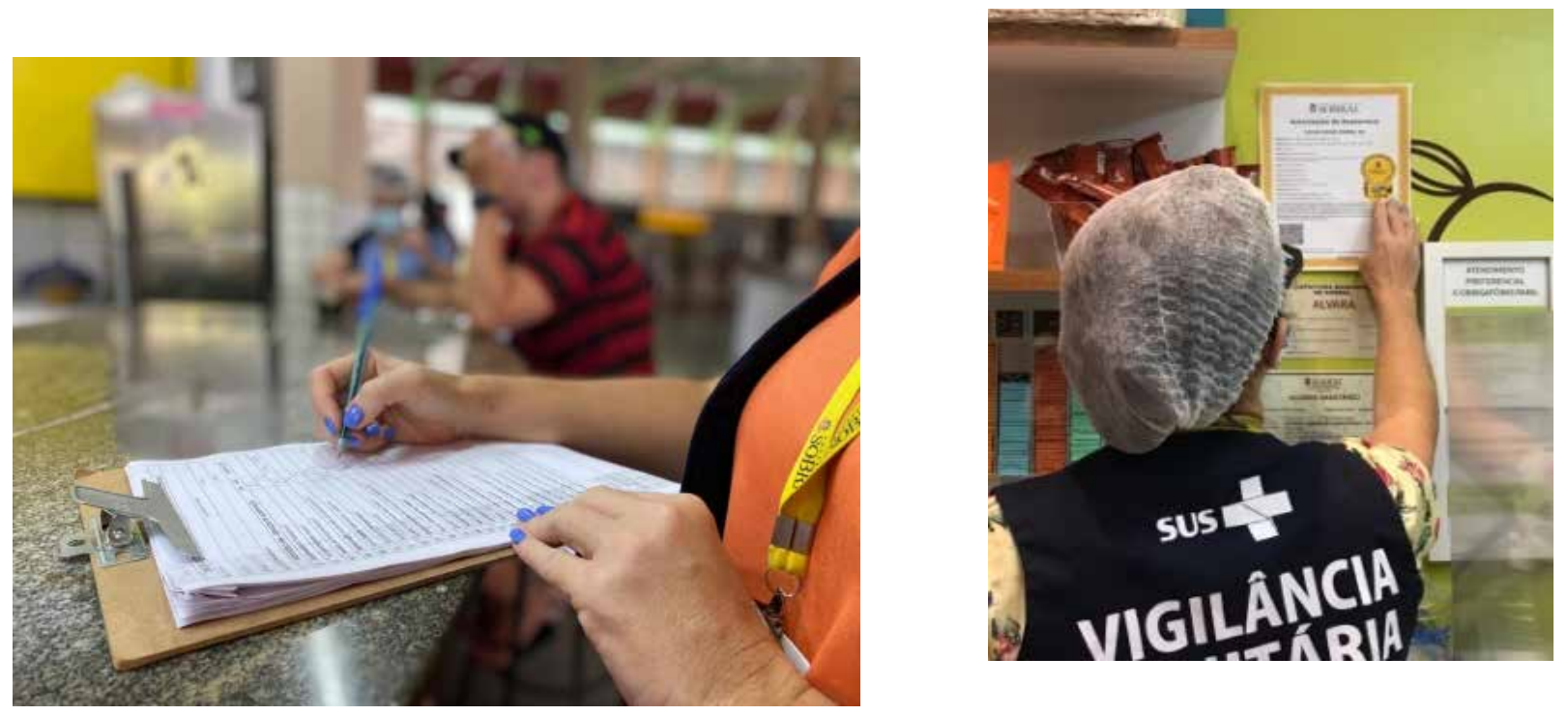

Fonte: Acervo fotográfico da Vigilância Sanitária. 
Figura 1 - Registro de inspeção, certificado de reabertura e selo de cumprimento das medidas sanitárias. Sobral, 2020. (continuação)
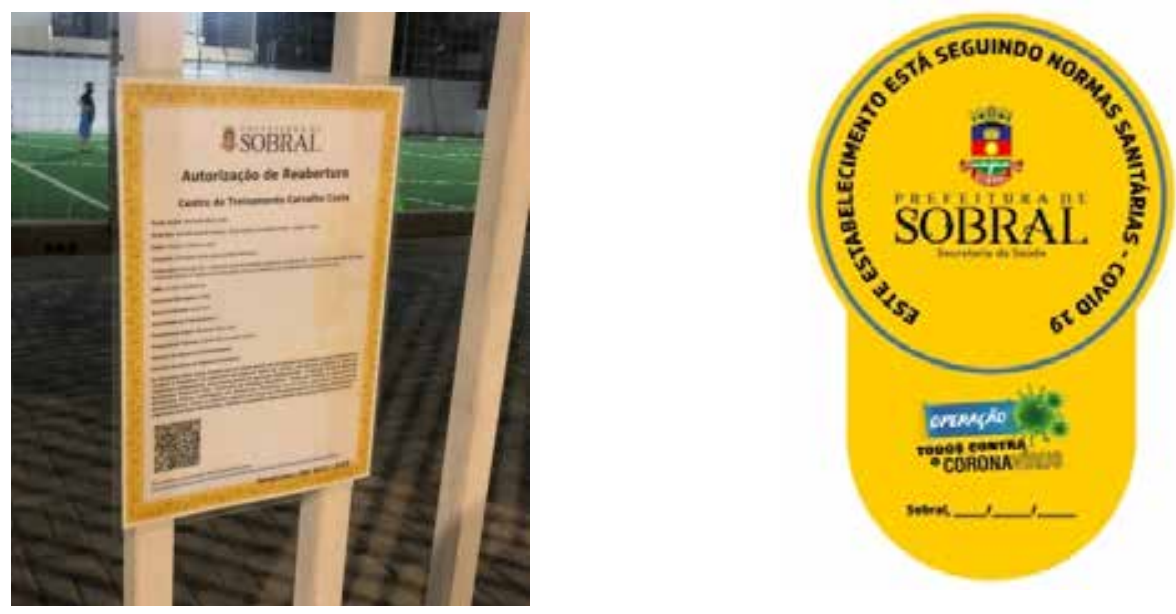

Fonte: Acervo fotográfico da Vigilância Sanitária.

Para cada endereço de funcionamento da empresa, deveria ser impresso um certificado, com seu respectivo protocolo. Em caso de qualquer descumprimento do Plano de Retomada, a Vigilância Sanitária poderia ser acionada.

Na página para a emissão do certificado (http://agendasol.sobral.ce.gov.br/solicitacao), o responsável pela empresa também poderia imprimir o material com as informações de ações comportamentais de prevenção ao novo coronavírus, caso não tivesse o seu próprio material gráfico. A comunicação visual foi um dos requisitos obrigatórios dos protocolos. Mesmo os estabelecimentos que não fecharam durante o período de isolamento social precisaram emitir o certificado. As empresas cadastradas com certificado de reabertura estão disponibilizadas para consulta pública no mapa de autorização de reabertura, através do link: http:// agendasol.sobral.ce.gov.br/relatorioAutorizacao.

Assim, a regulação sanitária deve buscar mecanismos eficazes de identificação, avaliação e redução de riscos, visando à segurança sanitária e à proteção da saúde da população. Vale, portanto, ressaltar a importância do princípio da precaução para o trabalho da VISA, segundo o qual os riscos potenciais de uma dada prática ou produto devem ser antecipados, visando ao desenvolvimento, em paralelo, de ações que busquem minimizar esses riscos. A observância do princípio de precaução é o compromisso ético que a VISA assume em prol da saúde e da equidade ${ }^{20}$.

Ressaltamos um desafio da regulação relacionado à publicação de normativas que tratam de forma extraordinária e temporária sobre os requisitos para a fabricação de dispositivos médicos identificados como prioritários para uso em serviços de saúde, em virtude da emergência de saúde pública internacional relacionada ao SARS-CoV-2.

0 risco de desabastecimento de produtos para a saúde e de equipamentos de proteção individual, usados pelos profissionais de saúde em razão da pandemia de Covid-19, exigiu das autoridades sanitárias do Brasil e de alguns países medidas rápidas para tentar evitá-lo e, ao mesmo tempo, preservar a manutenção de um padrão mínimo de qualidade e segurança dos produtos.

Em Sobral, exemplificamos uma empresa calçadista que mudou parte do seu processo produtivo para fabricar alguns produtos descartáveis como máscaras cirúrgicas e vestimentas hospitalares.

\section{Monitoramento dos casos suspeitos e confirmados de Covid-19 em descumprimento do isolamento social}

Entre as atividades demandadas à Vigilância Sanitária no enfrentamento da pandemia está o monitoramento dos casos suspeitos e confirmados de Covid-19 em descumprimento do isolamento social. A princípio, indagouse quem receberia essa responsabilidade. E, diante do inusitado, o que poderia ser feito para mitigá-lo? Tendo a VISA a definição legal de minimizar ou eliminar o risco, foi discutido e acordado que seria esse o serviço 
para o qual estava mais capacitada a oferecer o seu saber, na busca por intervir sobre a prática, em prol da população.

As clássicas formas de prevenção, de grande importância para a saúde pública, como o isolamento social e a adoção de bons hábitos de higiene da população, encontram barreiras para serem implementadas. A ação não fazia parte do cotidiano da VISA e, para que fosse colocada em prática, foi necessário o apoio de outros profissionais de saúde. A Secretaria Municipal de Saúde remanejou para a equipe da VISA uma técnica de enfermagem e uma fisioterapeuta; e a Escola de Saúde Pública Visconde Saboia, duas assistentes sociais da Residência Multiprofissional em Saúde da Família, para a ampliação da equipe e assegurar a abordagem multiprofissional.

Para o monitoramento, criou-se um fluxograma para padronizar a abordagem e o seguimento desses casos. Essa atividade se deu em parceria com a Coordenadoria da Atenção Primária (Figura 2). 0s pacientes suspeitos ou casos confirmados de Covid-19 eram acompanhados pela Estratégia Saúde da Família (ESF) para monitorar a evolução de sinais e sintomas. Os casos de descumprimento das orientações de isolamento, em geral, eram denunciados ao Plantão Epidemiológico, que funcionava 24 horas via WhatsApp, via telefone da VISA ou por meio da própria Equipe de Saúde da Família (eSF).

Após 0 recebimento da denúncia de descumprimento do isolamento social, a equipe tem a responsabilidade de verificar a veracidade e discutir com a equipe de monitoramento da Covid-19 da SMS se o caso em questão tem resultado de exame positivo e/ou a necessidade de quarentena baseada na notificação de casos. Verifica-se também se houve abordagem prévia pela eSF, seguida de avaliação do caso e definição se a abordagem é realizada no estabelecimento onde o paciente trabalha ou em sua residência, a depender do teor da denúncia.

Durante o período deste estudo, foram recebidas cerca de 180 denúncias relacionadas ao descumprimento do isolamento social; à não adesão ao uso de máscaras de proteção; ao não afastamento do trabalho nos casos suspeitos, e, em alguns casos, mesmo com confirmação do diagnóstico; e aos pacientes com Covid-19 apresentando sinais e sintomas de agravamento, mas que não aceitaram o encaminhamento hospitalar ou orientação de internação.

Foram realizadas 143 abordagens domiciliares

\section{"Durante o período deste estudo, foram recebidas cerca de 180 denúncias relacionadas ao descumprimento do isolamento social"}

e atendidas mais de 30 denúncias relacionadas aos trabalhadores com suspeita de Covid-19 que não foram afastados ou que voltaram antes do cumprimento da quarentena, bem como relacionadas à falta de medidas adotadas pelas empresas em relação aos cuidados e orientações dos contatos. Faz-se interessante destacar que, nos bairros que concentraram grande densidade populacional, os homens foram os que mais descumpriram a orientação de isolamento; e os idosos, a orientação dos encaminhamentos para Unidades Hospitalares.

0 estudo realizado por $\operatorname{Lima}^{20}$ et al. sobre aspectos comportamentais, valores e crenças da população cearense frente à pandemia de Covid-19 mostrou que alguns grupos estão mais vulneráveis à infecção, dentre eles: homens, indivíduos com baixa escolaridade, idosos a partir de 80 anos e aqueles residentes em municípios localizados no interior do estado. Esse resultado é reflexo, em grande parte, da minimização da propagação do vírus e do descumprimento de medidas recomendadas pelas autoridades sanitárias. $0 \mathrm{~s}$ idosos entrevistados estão cumprindo parcialmente a quarentena, pois acreditam que a pandemia não é uma ameaça "real" e que o clima cearense favorecerá a diminuição de casos da Covid-19. Curiosamente, os indivíduos que residem no interior do estado e/ou que possuem o Ensino Fundamental concluído também compartilham o mesmo pensamento. Ademais, verifica-se que o gênero masculino está associado à não realização voluntária da quarentena.

A abordagem domiciliar se mostrou uma estratégica importante, pois apresenta como aspecto positivo a aproximação dos profissionais ao contexto no qual os usuários estão inseridos, o que possibilita a valorização da dimensão subjetiva das práticas em saúde, das vivências dos usuários e dos trabalhadores, abrindo espaços de comunicação e diálogo entre saberes e práticas, além de novas perspectivas para a reflexão e ação $0^{21}$. 
Figura 2 - Fluxograma para monitoramento de casos suspeitos ou confirmados de Covid-19 em descumprimento do isolamento social. Sobral, 2020.

\section{Recebimento da denúncia}

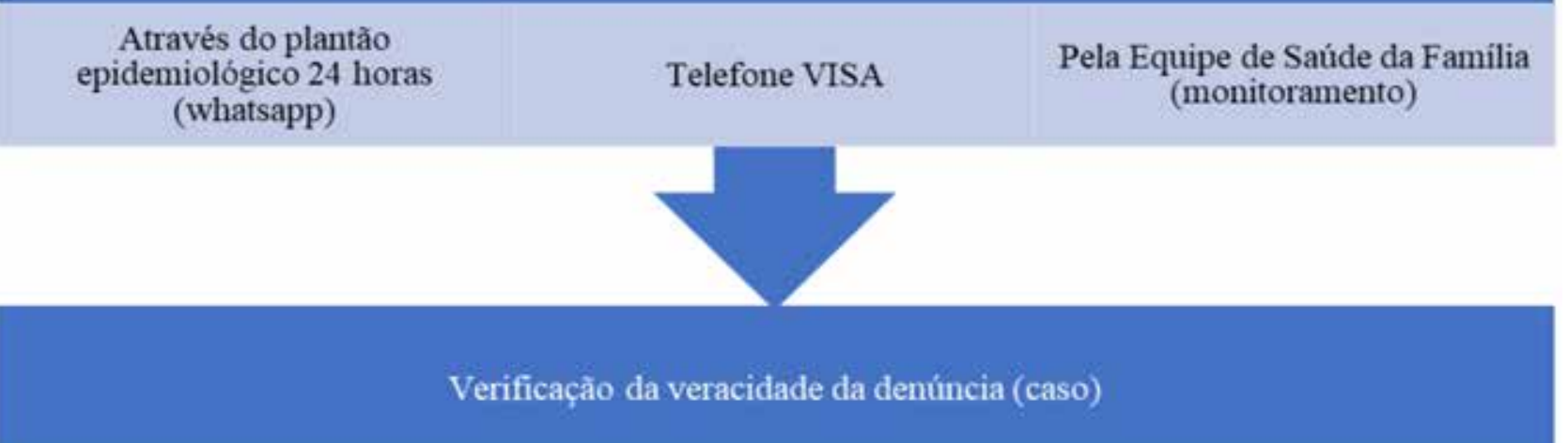

\section{Equipe de monitoramento se 0 caso tem resultado positivo, notificado e/ou necessidade de quarentena (dias)}
Organizar se a abordagem será domiciliar ou no ambiente de trabalho

Ver o acompanhamento da Equipe de Saúde da Família (se já realizada primeira abordagem)

\section{Realizaçăo da abordagem domiciliar ou no ambiente de trabalho}

Realiza a abordagem e entrega a notificação baseada na Lei $6437 / 77$. Decretos Municipais e Estaduais e artigos do Código Penal.

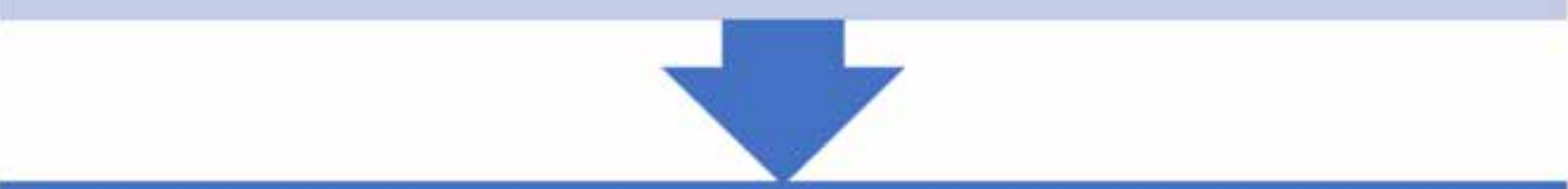

\section{Reincidência da denúncia}

Havendo uma nova denúncia relacionada ao mesmo caso e caracterizada a reincidência, o caso é encaminhado para a Coordenação da Atenção Primária

Fonte: Elaboração própria. 


\section{Informação, Comunicação e Educação para a Saúde}

As atividades desse eixo estão direcionadas ao fortalecimento da mudança da cultura sanitária junto à população e ao setor regulado, com as ações relacionadas à comunicação do risco sanitário; às orientações para o setor regulado, trabalhadores e população para a adoção de medidas de prevenção e controle de infecção humana pelo novo coronavírus (Covid-19); ao atendimento às demandas espontâneas da população (denúncias e/ou reclamações); à articulação com órgãos/instâncias de controle social e à articulação com as instituições de ensino para a inclusão de conteúdos relacionados às ações de VISA.

Para Costa, o direito à informação correta sobre os benefícios e os riscos dos bens relacionados à saúde integra o rol dos direitos do cidadão e do consumidor, portanto as ações da VISA não devem ser dirigidas apenas à fiscalização, que é fundamental, mas insuficiente. É necessário direcioná-las também aos cidadãos, divulgando informações adequadas e pertinentes, que contribuam na redução das assimetrias de informação e para subsidiar uma ação mais proativa e participativa do cidadão na defesa de seus direitos ${ }^{14}$.

Durante a pandemia, foi necessário repensar as estratégias de informação e diálogo com a população, junto aos profissionais e gestores da saúde, e com o setor regulado, a respeito das questões de Vigilância Sanitária. Para Lucchese 22 , há um grande espaço, precariamente explorado, para a ação educativa no âmbito da vigilância sanitária, tanto no que se refere ao entendimento do risco à saúde, envolvido nos atos e nas situações cotidianas, quanto no que se relaciona aos direitos da cidadania. A Vigilância Sanitária acopla a necessidade de dimensão educativa à possibilidade da ação punitiva formal.

Nas ações relacionadas à comunicação do risco sanitário, houve intensa participação em programas de rádio e TV, previamente gravados e com matérias ao vivo, assim como a produção de conteúdo para a publicação nas redes sociais. A comunicação do risco com a divulgação de informações que melhorem a consciência sanitária do setor regulado (indústria, comércio e serviços) e da população foi fundamental. As intervenções, que incluem ações comunicativas na defesa da saúde, contribuem para fortalecer as capacidades individuais e coletivas para lidar com os múltiplos e diversos condicionantes da saúde ${ }^{15}$ e
"Durante a pandemia, foi necessário repensar as estratégias de informação e diálogo com a população, junto aos profissionais $e$ gestores da saúde, e com o setor regulado"

a atuação do cidadão como parceiro do poder público no âmbito da VISA.

No atendimento às demandas espontâneas da população (denúncias e/ou reclamações), foram realizados cerca de 250 atendimentos com verificação in loco. 0 atendimento tem o objetivo de apurar os dados provenientes de denúncias e/ou reclamações de usuários, empresas e órgãos públicos (Ministério Público, por exemplo). Salienta-se que todas as denúncias/reclamações têm caráter sigiloso, sendo resguardada a identificação do usuário.

$\mathrm{Na}$ articulação com órgãos/instâncias de controle social, foram realizadas reuniões virtuais para a apresentação das ações e protocolos sanitários para o Conselho Municipal de Saúde e Conselho Municipal da Educação. Registra-se também a participação em atividades dos cursos de Medicina, Enfermagem, Odontologia e Residências Multiprofissionais em Saúde da Família e Saúde Mental, vinculados às instituições: Universidade Federal do Ceará, Universidade Estadual Vale do Acaraú, UNINTA e Escola de Saúde Pública Visconde de Saboia, com a finalidade de difundir conteúdos relacionados às ações da VISA, principalmente relativos ao contexto pandêmico.

Muitos fatores influenciam a resposta dos indivíduos às práticas de educação em saúde, o que pode causar conflitos e divergências nos saberes e nas práticas de saúde. Assim, a compreensão sobre a visão de mundo dos indivíduos, as crenças, os aspectos sociais, familiares e culturais representa um grande desafio para os profissionais que estão diretamente envolvidos no enfrentamento à pandemia pelo SARSCoV-2, seja nas práticas de cuidado, de vigilância, no planejamento ou na gestão em saúde ${ }^{23}$.

\section{CONCLUSÃO}

0s resultados dessa experiência demonstram a importância da criação de espaços de discussão entre os profissionais da Vigilância Sanitária e os demais 
trabalhadores das diversas áreas e setores, além de apontarem para o fato de que o fortalecimento da intersetorialidade se apresentou como uma estratégia viável na troca de conhecimentos entre os profissionais, promovendo uma reflexão crítica sobre o processo de trabalho da VISA no âmbito local.

0 contexto da pandemia trouxe aos profissionais da VISA muitas dificuldades; dentre elas, enfrentar a adaptação para o atendimento às novas demandas e o medo de se infectarem ou transmitirem a infecção aos membros da família. No entanto, a capacidade dos profissionais de reconhecer os obstáculos para a sua atuação profissional e de propor alternativas de melhorias em suas rotinas de trabalho resultou na maior capacidade de execução da ação local, ressignificando o processo de trabalho e propiciando uma maior visibilidade das ações pela população, setor regulado e gestão.

Por fim, o estudo reforça a importância de novas estratégias que constituam iniciativas voltadas para a superação de desafios históricos no processo de trabalho da Vigilância Sanitária, tornando o fazer mais integral e resolutivo, propondo uma articulação de políticas intersetoriais voltadas para a melhoria da saúde coletiva.

\section{CONTRIBUIÇÃO DOS AUTORES}

Verena Emmanuelle Soares Ferreira contribuiu com redação, análise e revisão crítica do manuscrito. Jéssica Maria Cavalcante Mesquita contribuiu com redação, análise e revisão crítica do manuscrito. Pollyana Dias Parente contribuiu com redação, análise e revisão crítica do manuscrito. Luiz Galdino da Costa Filho contribuiu com redação, análise e revisão crítica do manuscrito. Marília Gouveia Ferreira Lima contribuiu com redação, análise e revisão crítica do manuscrito. Alex Melo de Aguiar contribuiu com redação, análise e revisão crítica do manuscrito.

\section{REFERÊNCIAS}

1. Costa EA. Fundamentos da Vigilância Sanitária. In: Costa EA, organizadora. Vigilância Sanitária: Temas para Debate. Salvador: EDUFBA; 2009. p. 1136.

2. Brasil. Lei n. ${ }^{0} 8.080$, de 19 de setembro de 1990. Dispõe sobre as condições para a promoção, proteção e recuperação da saúde, a organização e o funcionamento dos serviços correspondentes e dá outras providências [document on the internet]. Diário Oficial da União, Brasília (1990 Sept 20 [cited 2020 Nov 12]; Sec 1. Available from: http://www.planalto.gov.br/ccivil 03/ leis/l8080.htm

3. Costa EAM. Vigilância Sanitária em Serviços de Saúde: os desafios da prática. VISA em debate [serial on the internet]. 2014 [cited 2020 Dec 03];2(2):2733. Available from: https://visaemdebate.incqs. fiocruz.br/index.php/visaemdebate/article/ view/148/118

4. Souza GS, Costa EA. Considerações teóricas e conceituais acerca do trabalho em vigilância sanitária, campo específico do trabalho em saúde. Cien Saúde Colet [serial on the internet]. 2010 Nov [cited 2020 Dec 10];15(Suppl 3):3329-40. Available from: http://www.scielo.br/scielo.php?script=sci arttext\&pid=S1413-81232010000900008\&lng=en

5. Oliveira WK, Duarte E, França GVA, Garcia LP. Como o Brasil pode deter a COVID-19. Epidemiol Serv Saúde [serial on the internet]. 2020 [cited $2020 \mathrm{Dec}$ 07];29(2):e2020044. Available from: http://www. scielo.br/scielo.php?script $=$ sci arttext\&pid $=$ S2237$\underline{96222020000200200 \& \operatorname{lng}=\text { en }}$

6. Szwarcwald CL, Souza JPRB, Malta DC, Barros MBA, Magalhães MAFM, Xavier DR, et al. Adesão às medidas de restrição de contato físico e disseminação da COVID-19 no Brasil. Epidemiol Serv Saúde [serial on the internet]. 2020 [cited $2020 \mathrm{Dec}$ 06];29(5):e2020432. Available from: http://www. scielo.br/scielo.php?script=sci arttext\&pid=\$223796222020000500305\&lng =en

7. Porto MF. No meio da crise civilizatória tem uma pandemia: desvelando vulnerabilidades e potencialidades emancipatórias. VISA em debate [serial on the internet]. 2020 May [cited 2020 Dec 05];8(3):2-10. Available from: http://www. visaemdebate.incqs.fiocruz.br

8. Instituto Brasileiro de Geografia e Estatística. Cidades e Estados [home-page on the internet]. 2020 [cited 2020 Dec 03]. Available from: https:// www.ibge.gov.br/cidades-e-estados

9. Sobral. Decreto n. ${ }^{0} 2.369$ de 13 de março de 2020. Declara estado perigo público iminente [document on the internet]. Diário Oficial do Município, Sobral (2020 Mar 13 [cited 2020 Dez 12]). Available from: http://www.sobral.ce.gov. br/diario/public/files/diario/4f532ab2361eeec e2b08d4dde8012de1.pdf

10. Sobral. Decreto n. ${ }^{0} 2.371$, de 16 de março de 2020. Decreta estado de emergência no âmbito do município de sobral e estabelece medidas para enfrentamento do novo coronavírus (COVID 19) [document on the internet]. Diário Oficial do Município, Sobral (2020 Mar 16 [cited 2020 Dez 12]). Available from: http://transparencia. sobral.ce.gov.br/arquivo/nome:4aebebdd164a be7bcb5f3b3c7c8382c1.pdf 
11. Soares CHA, Linhares MSC. Vigilância à Saúde no Município de Sobral-CE: Aspectos históricos, conceituais e atuação na Saúde Pública. Sanare [serial on the internet]. 2008 [cited 2020 Dec 05];7(1). Available from: https://sanare.emnuvens. com.br/sanare/article/view/48

12. Brasil. Portaria $n^{0} 1.399$, de 15 de dezembro de 1999. Regulamenta a NOB SUS $01 / 96$ no que se refere às competências da União, estados, municípios e Distrito Federal, na área de epidemiologia e controle de doenças, define a sistemática de financiamento e dá outras providências [document on the internet]. Brasília: Ministério da Saúde [cited 2020 Dez 12]. Available from: http://www.funasa.gov.br/site/wpcontent/files mf/Pm 1399 1999.pdf

13. Sobral. Decreto n. ${ }^{0} 2346$, de 07 de fevereiro de 2020. Altera a estrutura organizacional e aprova 0 regulamento, a distribuição e a denominação doscargos de provimento em comissão da secretaria municipal da saúde, e dá outras providências. Diário Oficial do Município, Sobral (2020 Mar 13 [cited 2020 Dez 12]). Available from: http://www.sobral.ce.gov.br/ d i a r i o/public/files/diario/ b3e7a48e267a52df251538c399668d13.pdf

14. Costa EA. Regulação e Vigilância Sanitária: Proteção e defesa da Saúde. In: Rouquayrol MZ, Gurgel M, organizadores. Rouquayrol - Epidemiologia \& Saúde. 7. ed. Rio de Janeiro: MedBook; 2013. p. 493-520.

15. Silva JAA, Costa EA, Lucchese G. SUS 30 anos: Vigilância Sanitária. Cien Saúde Colet [serial on the internet]. 2018 [cited 2020 Dec 02];23(6):1953-61. Available from: http://www. scielo.br/scielo.php?script=sci arttext\&pid $=S 1413-$ $\underline{81232018000601953 \& \operatorname{lng}=\text { en }}$

16. Costa FC, Kobayashi LPM. A Vigilância Sanitária no Sistema Único de Saúde: Trajetória e área de atuação [monografia]. Londrina: Centro Universitário Filadélfia - UniFil; 2012.

17. Brasil. Política Nacional de Promoção da Saúde. Textos Básicos de Saúde. 3. ed. Brasília (DF): Ministério da Saúde; 2010. Série B.

18. Moraes EN, Viana LG, Resende LMH, Vasconcellos LS, Moura AS, Menezes A, et al. COVID-19 nas instituições de longa permanência para idosos: estratégias de rastreamento laboratorial e prevenção da propagação da doença. Cien Saúde Colet [serial on the internet]. 2020 Sep [cited 2020 Dec 04];25(9):3445-58. Available from: http://www.scielo.br/scielo.php?script=sci arttext\&pid $=$ S1413-81232020000903445\&lng =en

19. Sobral. Prefeitura Municipal de Sobral. Plano de retomada das atividades produtivas em Sobral e respectivas regras de flexibilização do distanciamento social em razão da pandemia -
COVID-19. Sobral (CE); 2020.

20. Lima DLF, Dias AA, Rabelo RS, Cruz ID, Costa SC, Nigri FMN, et al. COVID-19 no estado do Ceará, Brasil: comportamentos e crenças na chegada da pandemia. Cien Saúde Colet [serial on the internet]. 2020 May [cited 2020 Dec 04];25(5):1575-86. Available from: http://www.scielo.br/scielo.php?script=sci arttext\&pid=S1413-81232020000501575\&Lng=en

21. Paim JS, Almeida Filho N. Saúde coletiva: uma "nova saúde pública" ou campo aberto a novos paradigmas? Rev Saúde Pública [serial on the internet]. 1998 Aug [cited 2020 Dec 05];32(4): 299-316. Available from: http://www.scielo. br/scielo.php?script $=$ sci arttext\&pid $=S 0034-$ $\underline{89101998000400001 \& \operatorname{lng}=\text { en }}$

22. Lucchese G. A Vigilância Sanitária no Sistema Único de Saúde. In: De Seta MH, Pepe VLE, Oliveira G0, organizadores. Gestão e Vigilância Sanitária: modos atuais do pensar e fazer [livro eletrônico]. Rio de Janeiro: Fiocruz; 2006. p. 33-48.

23. Palácio MAV, Takenami I. Em tempos de pandemia pela COVID-19: o desafio para a educação em saúde. Revista Vigilância Sanitária em Debate: Sociedade, Ciência \& Tecnologia. VISA em debate [serial on the internet]. 2020 [cited 2020 Dec 04];8(2):1015. Available from: http://www.visaemdebate.incqs. fiocruz.br

24. Sobral. Decreto n. ${ }^{0} 2.456$, de 28 de junho de 2020. Prorroga o isolamento social rígido no município de Sobral, e dá outras providências. Diário Oficial do Município, Sobral (2020 June 28 [cited 2020 Dec 17]). Available from: http://www.sobral.ce.gov.br/media/com download/files/20200628194512.pdf

25. Chagas MF, Villela WV. Vigilância Sanitária e promoção de saúde: apontamentos para além da regulação e controle. VISA em debate [serial on the internet]. 2014 [cited 2020 Dec 17];2(3):306. Available from: http://www.visaemdebate.incqs. fiocruz.br

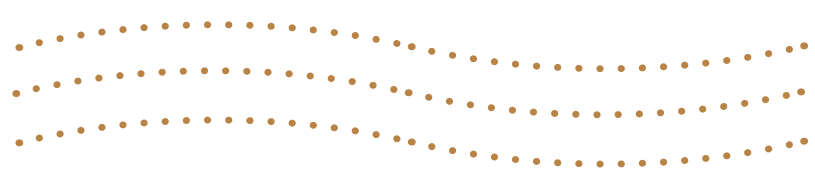

